\title{
ANTIDIABETIC ACTIVITY OF SENNA SURATTENSIS IN ALLOXAN-INDUCED DIABETIC RATS
}

\author{
ELLAPPAN THILAGAM ${ }^{1 *}$, KUMARAPPAN CHIDAMBARAM ${ }^{2}$, SUBHASH CHANDRA MANDAL ${ }^{1}$ \\ ${ }^{1}$ Department of Pharmaceutical Technology, Pharmacognosy and Phytotherapy Laboratory, Jadavpur University, Kolkata, West Bengal, \\ India. ${ }^{2}$ Department of Pharmacology and Toxicology, School of Pharmacy, King Khalid University, Saudi Arabia. \\ Email: ctkumarrx@gmail.com \\ Received: 13 November 2017, Revised and Accepted: 27 December 2017
}

ABSTRACT

Objective: Senna surattensis is a shrub plant which has been known for its diverse biological and pharmacological properties. This study is aimed to evaluate the antidiabetic activity of ethanolic extracts of $S$. surattensis (EESS) leaves in alloxan-induced diabetic rats.

Methods: Experimental diabetes was induced by injection of a single dose of alloxan (120 mg/kg, intraperitoneal). Adult male Wistar albino rats were divided into five groups; normal control, diabetic control, diabetic EESS ( $200 \mathrm{mg} / \mathrm{kg}$ body weight (bw), diabetic EESS ( $400 \mathrm{mg} / \mathrm{kg}$ bw), and diabetic glibenclamide ( $5 \mathrm{mg} / \mathrm{kg} \mathrm{bw}$ ). Extracts were treated concurrently for 21 days. Blood samples were collected and centrifuged for estimation of fasting blood glucose (FBG), bw, serum biomarkers, lipid profile, total protein, albumin, and glycosylated hemoglobin (HbA1C) contents.

Results: The increase in FBG, bw, liver biomarkers serum glutamic oxaloacetic transaminase, serum glutamic pyruvic transaminase, alkaline phosphatase, free fatty acid, phospholipids (PL), triglycerides, total cholesterol, low-density lipoprotein cholesterol, high-density lipoprotein cholesterol, total protein, albumin, and HbA1C content were recorded in diabetic control rats. Daily oral administration of EESS treatment significantly $(\mathrm{p}<0.01)$ reverted the levels of serum biomarkers and enzymes activities to near normal values. A similar reduction was produced in FBG after 21 days of extract administration which compared significantly $(\mathrm{p}<0.01)$ with the control group and glibenclamide treated groups.

Conclusion: The results suggest that EESS has anti-diabetic activity in diabetic rats, thereby justifying its traditional claim and augmenting it into the present system of medicine.

Keywords: Senna surattensis, Alloxan, Hyperglycemia, Diabetes mellitus, Hypolipidemic.

(C) 2018 The Authors. Published by Innovare Academic Sciences Pvt Ltd. This is an open access article under the CC BY license (http://creativecommons. org/licenses/by/4. 0/) DOI: http://dx.doi.org/10.22159/ajpcr.2018.v11i4.23632

\section{INTRODUCTION}

Diabetes mellitus is a group of metabolic disorder is characterized by hyperglycemia and disturbances of carbohydrate, lipid, and protein metabolisms. Long-term hyperglycemia during diabetes causes glycation of body proteins that lead to secondary complications affecting kidney, eye, nerve, and arteries. It is considered as one of the five leading causes of death in the world [1]. Altogether diabetes has shadowed the spread of modern lifestyle, and it can be associated to surge overweight and sedentary inhabitants [2]. The global problem of diabetes mellitus stances massive social expenses and has chief implications for all healthcare structures. Diabetic dyslipidemia results in accumulation of excess free fatty acids (FFA), which are converted to triglycerides (TGs) in the liver. The consequence of fat accumulation is increased small dense low-density lipoprotein cholesterol (LDLc) and TGs levels and decreased high-density lipoprotein cholesterol (HDLc), which contributes to cardiovascular risk in diabetes. It is now well-known that the hyperlipidemia signifies a major risk factor for the premature development of diabetes and its complications.

Experimental models using animal provide clear clues for the understanding of the molecular and pathological state of diabetes mellitus and are valuable for the screening of drugs for the prevention and management of diabetes. The pathophysiology of diabetes involves a very complex cascade of several interrelated mechanisms. Alloxan is a universally used chemical to produce experimental diabetic animals in the labs for its ability to damage insulin-producing beta cells. It is generally accepted that free radicals generated by alloxan cause beta cell injury that is key to its part as a diabetogenic agent. As it has been widely accepted that alloxan selectively destroys the insulin-producing beta cells found in the pancreas; hence, it is used to induce diabetes in laboratory animals.
There is increasing demand by patients to use natural products with anti-diabetic activity due to side effects associated with the use of oral hypoglycemic agents. Many of the currently available oral hypoglycemic drugs possess a number of serious toxic effects [3]. Meanwhile, the management of diabetes mellitus without adverse effects is still a major challenge. Dietary involvement, mostly the practice of traditional medicine derived from natural sources, is a major strength in the management of diabetes [4].

Traditionally, a number of plants have been used in various herbal preparations in the management of diabetes and only a few of them have been proven scientifically. Plant drugs are considered to be less toxic and free from side effects than synthetic ones [5]. Senna surattensis. (Caesalpiniaceae) is commonly known as Glaucous cassia and distributed throughout India. It is commonly used in folk medicine as antihyperglycemic for the management of diabetes mellitus [6,7]. The plant is also known for its use in gonorrhea blennorrhea and jaundice. Bark, aerial parts and leaves are useful in for the management of diabetes and gonorrhea [8]. The plant found to contain anthraquinone, flavonol glycosides, chrysophanol, physcion, kaempferide, and quercetin [9]. The extracts of $S$. surattensis have been shown to have antimicrobial, antihyperlipidemic, antioxidant, and hepatoprotective activities [10-13]. We have also previously demonstrated the hypoglycemic effect of ethanolic extracts of $S$. surattensis (EESS) using in vitro models of diabetes mellitus [14]. Literature surveys have yielded scanty information on the pharmacological properties of $S$. surattensis for diabetes management. However, no systematic study was carried out on the leaf extracts of $S$. surattensis for its in vivo anti-diabetic activity using an animal model. Hence, the present study first time aimed to investigate the anti-diabetic activity of leaf extracts of $S$. surattensis using alloxan treated diabetic rats to ascertain the scientific basis for the use in the treatment of diabetes mellitus. Here, the antihyperglycemic 
activity of EESS is evaluated in detail with scientific approach including its effects on biochemical parameters.

\section{METHODS}

\section{Plant materials}

Fresh leaves of $S$. surattensis were collected from Tiruchirappalli (Tamil Nadu, India) in December 2006 and authenticated by the Botanical Survey of India (Coimbatore, Tamil Nadu, India; Ref. No.: BSI/SC/5/23/06-07/Tech-1638). An authentic voucher specimen was deposited in the Herbarium Division of Pharmacognosy, Department of Pharmaceutical Technology, Jadavpur University, Kolkata, India.

\section{Preparation of plant extract}

The collected leaves of S. surattensis were air dried at room temperature without exposure to sunlight, coarsely powdered. $300 \mathrm{~g}$ of the powdered leaf was packed in Soxhlet apparatus and extracted with ethanol $(95 \%)$. The solvent was then evaporated under reduced pressure in a rotary evaporator (Superfit, India) at $<40^{\circ} \mathrm{C}$ to obtain a dry extract (yield $24.25 \% \mathrm{w} / \mathrm{w}$ ) that was stored at $-20^{\circ} \mathrm{C}$ in a refrigerator until further use. The extracts were subjected to a qualitative test for the identification of various phytochemical constituents as per the standard procedures [15]. The dose of each extract was calculated according to body weight (bw) before administration to the diabetic rats.

\section{Animals and ethical approval}

Male Wistar albino rats weighing about 250-300 g bw were used in the present study (M/S Ghosh Enterprises, Kolkata, India). The animals were collected from a breeding colony they were housed in polypropylene cages and fed with commercial diet from Hindustan Lever Ltd. (Bangalore, India) and had free access to water ad libitum during the experiment. The animals were acclimatized to the laboratory condition (temperature $21 \pm 2^{\circ} \mathrm{C}$ ) with a light period $(7.00$ a.m to 7 p.m. and relative humidity 55-70\%) for 2 weeks before the start of the experiment. The experiments were performed complied with the rulings of the Committee for the Purpose of Control and Supervision of Experiments on Animals (CPCSEA) New Delhi, India (Registration No: 0367/01/C/CPCSEA), and the study was permitted by the Institutional Animal Ethical Committee (IEAC) of the Jadavpur University, Kolkata.

\section{Induction of experimental diabetes mellitus}

Diabetes was induced by a single intraperitoneal injection of alloxan monohydrate $(150 \mathrm{mg} / \mathrm{kg} \mathrm{bw})$ in a freshly prepared sterile normal saline solution [16]. Diabetes was confirmed by measuring fasting blood glucose (FBG) level $72 \mathrm{~h}$ after alloxan injection only rats with glucose level $>300 \mathrm{mg} / \mathrm{dl}$ as well as with polydipsia, polyuria, and polyphagia was selected for the experiment.

\section{Experimental design}

In this study, a total of 30 rats (24 diabetic surviving rats and 6 normal rats) were used. The rats were divided into five groups $(n=6)$. The Group 1 (non-diabetic control) and Group 2 (diabetic control) rats received 2\% Tween $80(2 \mathrm{ml} / \mathrm{kg}$ bw), while Group 3 and 4 diabetic rats treated with EESS $(200 \mathrm{mg} / \mathrm{kg}$ and $400 \mathrm{mg} / \mathrm{kg}$ bw) once a day using the intragastric tube for 21 days. Group 5 diabetic rats were treated with standard drug glibenclamide ( $5 \mathrm{mg} / \mathrm{kg}$ bw). On day 21, blood was collected from the retro-orbital flexus using microcapillary tubes with mild ether anesthesia from overnight fasted rats. The blood was collected with or without ethylenediaminetetraacetic acid. The blood samples were kept at room temperature for $1 \mathrm{~h}$, and serum was separated after centrifugation at $3000 \mathrm{rpm}$ for $10 \mathrm{~min}$ for lipid estimation.

\section{Estimation of biochemical parameters}

FBG was measured by ACCU-Chek Touch Glucometer (Accu-Chek, Roche Diagnostic, USA). Changes in bw were estimated at the end of experiments. Serum biomarkers such as serum glutamic oxaloacetic transaminase (SGOT), serum glutamic pyruvic transaminase (SGPT), alkaline phosphatase (ALP), FFA, PLs, HbA1C, HDLc, LDLc, TGs, TC, total protein, and albumin were measured by spectrophotometrically (Spekol 1200, Japan) using Span diagnostic Kits (Mumbai, India).
Statistical analysis

Data were statistically evaluated using one-way analysis of variance, followed by Dunnett's t-test using GraphPad InStat Statistical software (San Diego, CA, USA). The values were considered significant when $\mathrm{p}<0.01$ and $\mathrm{p}<0.05$.

\section{RESULTS}

\section{Effect on FBG and bw}

Changes in FBG and bw in diabetic control and EESS treatment were presented in Figs. 1 and 2. In this study, alloxan-induced diabetic rats showed significant $(\mathrm{p}<0.01)$ reduction in bw. FBG levels of the diabetic control rats were higher than those of normal rats. Administration of EESS (200 and $400 \mathrm{mg} / \mathrm{kg}$ ) and glibenclamide ( $5 \mathrm{mg} / \mathrm{kg}$ ) significantly $(p<0.01)$ increased the bw within 21 days. After administration of EESS a significant $(p<0.01)$ dose-dependent decrease in FBG levels was observed compared with diabetic control group.

\section{Effect on serum liver profile}

Fig. 3 showed the liver biomarker such as SGOT, SGPT, and ALP in the serum of control and experimental groups. Serum transaminases SGOT, SGPT, and ALP levels were significantly increased in the diabetic control rats. After treatment with EESS $(200 \mathrm{mg} / \mathrm{kg}$ and $400 \mathrm{mg} / \mathrm{kg})$ and glibenclamide ( $5 \mathrm{mg} / \mathrm{kg})$, the serum transaminases level was brought back to almost near normal levels $(\mathrm{p}<0.01)$.

\section{Effect on serum lipid profile}

The level of FFAs, PLs, TC, TGs, HDLc, and LDLc in the serum of EESS treated and control diabetic rats are presented in Fig. 4. Significant

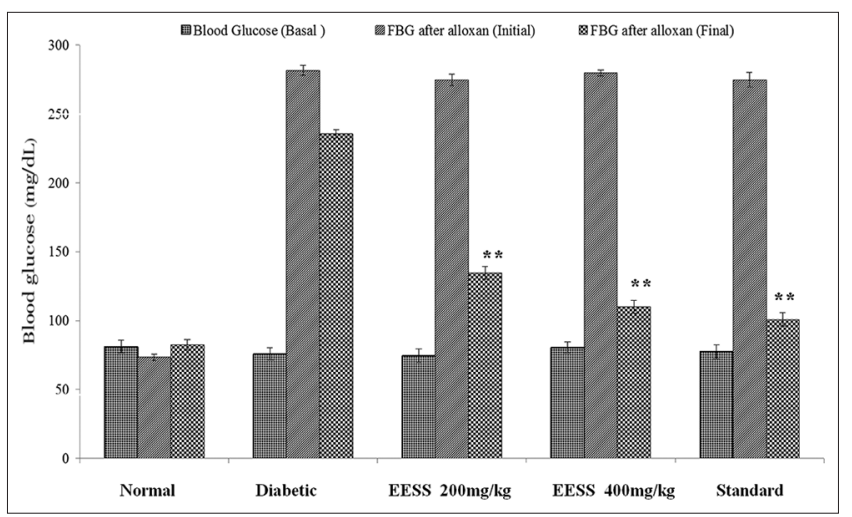

Fig. 1: Effect of ethanolic extracts of $S$. surattensis on fasting blood glucose in alloxan-induced diabetic rats. Values are expressed as mean \pm standard error mean of six animals (error bars in the figure). ${ }^{* *} \mathrm{p}<0.01$ versus control group (before treatment)

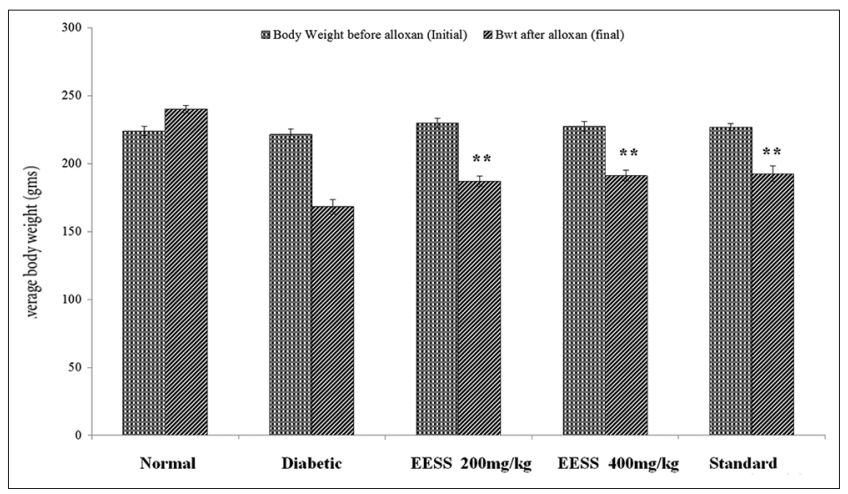

Fig. 2: Ethanolic extracts of $S$. surattensis on body weight in alloxan-induced diabetic rats. Values are expressed as mean \pm standard error mean of six animals (error bars in the figure). ${ }^{* *} \mathbf{p}<0.01$ versus control group (before treatment) 
reductions in serum HDLc were observed in alloxan-induced diabetic rats when compared to control rats (Group 1). On administration of EESS $(200 \mathrm{mg} / \mathrm{kg}$ and $400 \mathrm{mg} / \mathrm{kg}$ ) for 21 days to the diabetic rats, serum HDLc levels were found to be restored in normal. Furthermore, the fatty acids, phospholipids (PL), total cholesterol (TC), TGs, and LDLc levels were elevated significantly in alloxan-induced control diabetic rats compared to control rats. Both the doses of EESS and glibenclamide treatment significantly $(\mathrm{p}<0.01)$ reduced above parameters compared to diabetic control rats.

\section{Effect on total protein, albumin, and HbA1C}

Figs. 5 and 6 showed the effect of EESS and glibenclamide on total protein and albumin in control and alloxan diabetic rats. Significant reductions in serum protein and albumin were observed in alloxaninduced diabetic rats (Group 2) when compared to control rats (Group 1). On administration of EESS to the diabetic rats, serum protein albumin and protein levels were found to be restored in normal. Furthermore, the HbA1C level was elevated significantly in alloxaninduced diabetic rats compared to control rats. Both the doses of EESS and glibenclamide treatment significantly reduced $\mathrm{HbA} 1 \mathrm{C}$ compared to diabetic control rats.

\section{DISCUSSION}

Diabetes mellitus is perhaps the fastest-developingmetabolic disorder in worldwide, which raises the need for more challenging and appropriate treatments. Traditional medicinal plant approaches have been used for many centuries in the treatment of diabetes. In India, S. surattensis leaves are widely used in folk medicine by diabetic patients to attenuate

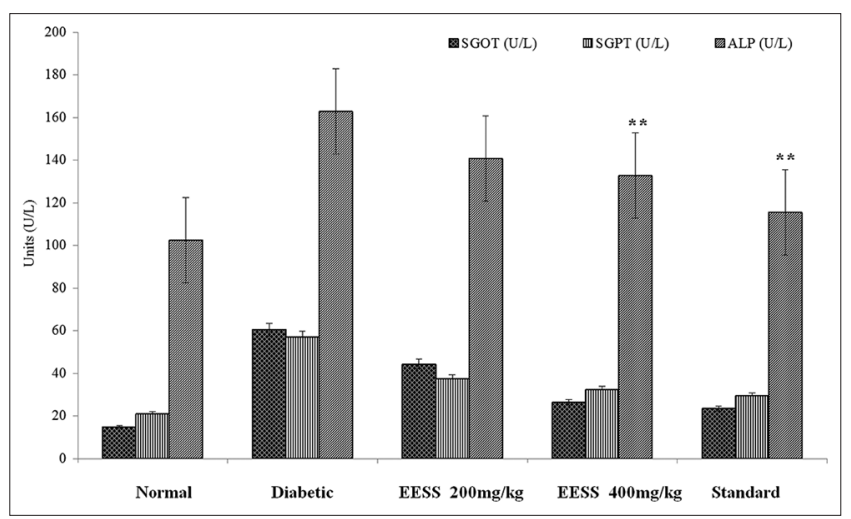

Fig. 3: Effect of ethanolic extracts of $S$. surattensis serum liver profiles in alloxan-induced diabetic rats. Values are expressed as mean \pm standard error mean of six animals (error bars in the figure). ${ }^{* *} \mathbf{p}<0.01$ versus control group (before treatment)

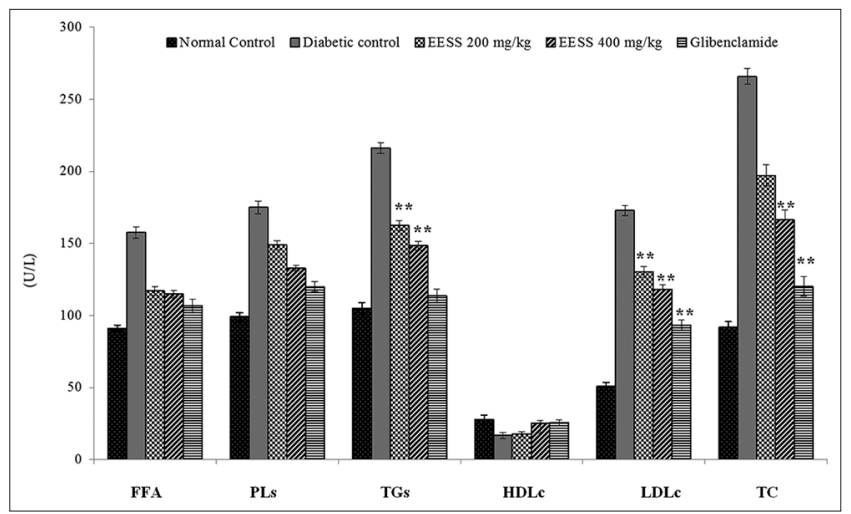

Fig. 4: Effect of ethanolic extracts of $S$. surattensis on serum lipids levels in alloxan-induced diabetic rats. Values are expressed as mean \pm standard error mean of six animals (error bars in the figure). ${ }^{* *} \mathbf{p}<0.01$ versus control group (before treatment) hyperglycemia caused by diabetes mellitus. This plant has been traditionally used in many countries as food products and for medicinal uses. S. surattensis flowers and leaves have been studied extensively, and the therapeutic properties such as antioxidant, hepatoprotective, and antimicrobial and have been reported $[17,18]$. The phytochemical analysis has shown the presence of potent phytochemicals such as flavonoids, terpenoids, glycosides, steroids, saponin, and phenols. It has long been used to treat diabetes mellitus and related hyperlipidemia. However, its pharmacological bases are not well understood in animal models of diabetes mellitus. The results of this study indicated that EESS not only possessed significant hypoglycemic effect but also had remarkable hypolipidemic effect in alloxan-induced diabetic rats.

In this study, the administration of alloxan, as expected, caused by significant hyperglycemia and elevated serum lipid levels. Alloxan, a cytotoxic agent, causes a condition of insulin-dependent diabetes through its ability to induce reactive oxygen species formation, leading to selective necrosis of the pancreatic beta cells $[19,20]$. Thus, alloxan injection results in pancreas $\beta$-cell death and histological changes then leads to the decrease of serum insulin level and elevation of glucose level. Before EESS treatment of alloxan-induced diabetic rats, the substantial rise in FBG was associated with increases in TC and TGs and decrease of HDLc. After EESS treatments, FBG, TC, and TGs of all tested rats were significantly decreased, and at the same time, HDLc was increased. Hyperlipidemia is one of the major cardiovascular risk

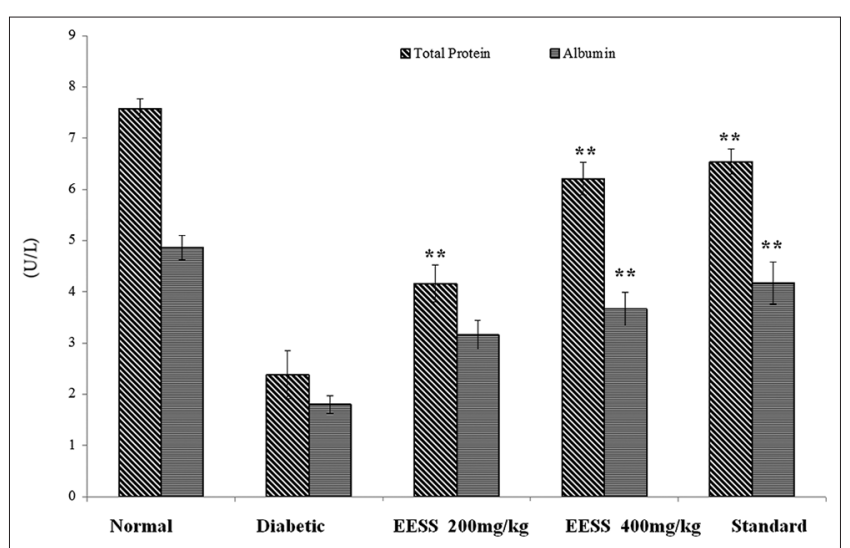

Fig. 5: Effect of ethanolic extracts of $S$. surattensis on serum protein levels in alloxan-induced diabetic rats. Values are expressed as mean \pm standard error mean of six animals (error bars in the figure). ${ }^{* *} \mathbf{p}<0.01$ versus control group (before treatment)

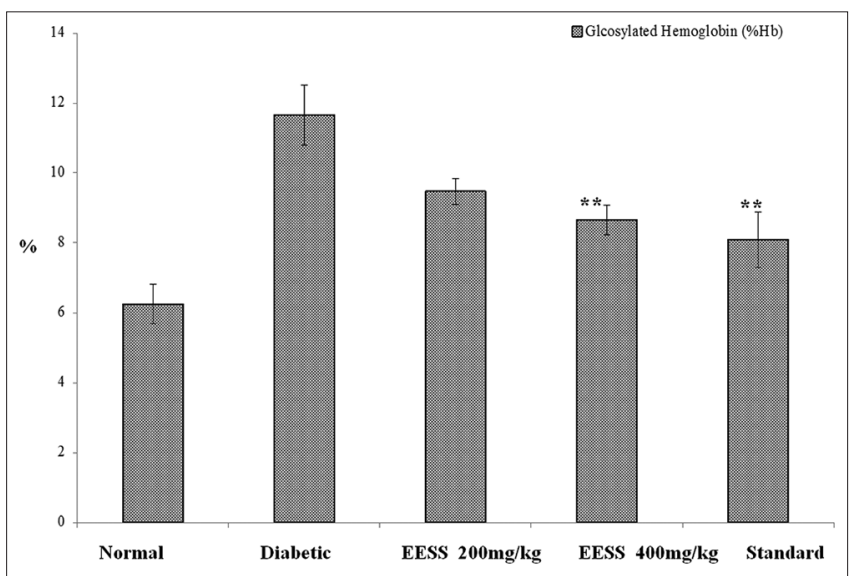

Fig. 6: Effect of ethanolic extracts of $S$. surattensis on HbA1C $(\% \mathrm{Hb})$ in alloxan-induced diabetic rats. Values are expressed as mean \pm standard error mean of six animals (error bars in the figure). ${ }^{* *} \mathbf{p}<0.01$ versus control group (before treatment) 
factors. Diabetes is associated with profound changes in serum lipid levels which increase the risk of metabolic syndrome [21]. Prolonged oral administration of EESS for 3 weeks ensued in major progress of serum lipid profile in alloxan-treated diabetic rats. It is well known that hyperglycemia is the main factor of plasma VLDLc and TGs [22], so the strong hypolipidemic effect of EESS could also be facilitated by the improvement of hyperglycemia.

$\mathrm{HbA1C}$ is considered as an important diagnostic marker and helps to know about the degree of protein glycation, long-term blood sugar level and correlation of diabetes-associated complications. HbA1C is abnormally high in diabetes, with chronic hyperglycemia and reflect their metabolic control $[23,24]$. HbA1C was found to be increased in patients with diabetes mellitus for approximately $16 \%$, and the amount of increase was found directly proportional to the FBG level. In this study, alloxan-induced diabetic rats showed a significant increase $(p<0.01)$ HbA1C level compared with normal rats. The ethanol extract of $S$. surattensis leaf treated rats showed a significant decrease $(\mathrm{p}<0.01)$ in the content of HbA1C that could be due to an improvement in glycemic status.

The decrease in bw in diabetic as clearly shows a loss or degradation of structural proteins due to diabetes. The ability of the EESS to protect from maximum bw loss seems to be due to its ability to reduce hyperglycemia. The decrease in TP and albumin may be due to microproteinuria and albuminuria, which are important clinical markers of diabetic nephropathy, and/or may be due to increased protein catabolism [25]. The results of the present study confirmed significant raise in TP and albumin levels to be similar to their normal levels after the treatment of the diabetic rats with EESS. Furthermore, elevated serum SGOT, SGPT, and ALP levels were described in diabetes mellitus, and it may be due to liver dysfunction [26]. In this study, an elevated level of SGOT, SGPT, and ALP was observed in alloxan-induced diabetic rats which may have occurred by leakage of enzymes from the liver cytosol into the bloodstream; it represents the toxicity of alloxan on the liver. Diabetic rats treated with EESS significantly decreased SGOT, SGPT, and ALP levels, which signify the protective effect of EESS in alloxan-treated diabetic rats. Moreover, it was reported earlier that the extract of $S$. surattensis did not show any signs of toxicity based on acute oral toxicity studies [27].

\section{CONCLUSION}

Diabetes mellitus has been a serious metabolic disorder all over the world. Medicinal plants play a key role in the treatment of diabetes due to their active constituents. Current results have confirmed that polyphenolic compounds and flavonoids are major bioactive components of the hypoglycemic effect of EESS. However, their detailed mechanism of action needs further investigation. In summary, the present study has shown that $S$. surattensis extracts validate clears hypoglycemic and hypolipidemic effects in alloxan-induced diabetic rats. This study is supportive for understanding mechanism of action of EESS, and also exposes the potential of S. surattensis for use as a natural oral hypoglycemic agent with hypolipidemic effects. However, longer duration of chronic studies is necessary to elucidate the exact mechanism of action so as to develop it as a potent anti-diabetic drug.

\section{ACKNOWLEDGMENTS}

The authors are grateful to All India Council for Technical Education (New Delhi, India) for providing financial support through its Quality Improvement Scheme (QIP).

\section{REFERENCES}

1. Sharma S, Chaturvedi M, Edwin E, Sagrawat H. Evaluation of the phytochemicals and antidiabetic activity of Ficus bengalensis. Int $\mathrm{J}$ Diab Dev 2007;27:57-9.
2. Rakesh KV, Vats RK, Kumar V, Abhishek K, Alka M, Uma R. Emerging targets for diabetes. Curr Sci 2003;88:241-9.

3. Zhang BB, Moller DE. New approaches in the treatment of Type 2 diabetes. Curr Opin Chem Biol 2000;4:461-7.

4. Rahimi R, Nikfar S, Larijani B, Abdollahi M. A review on the role of antioxidants in the management of diabetes and its complications. Biomed Pharmacother 2005;59:365-73.

5. Eidi A, Eidi M, Esmaeili E. Antidiabetic effect of garlic (Allium sativum L.) in normal and streptozotocin-induced diabetic rats. Phytomedicine 2006;13:624-9.

6. Singh V. Monograph on Indian Subtribe Cassiinae (Caesalpinaceae). New Delhi: Pawankumar Scientific Publisher; 2001. p. 215-9.

7. Tripathi YC, Pratibha S, Devesh T. Phytochemical evaluation and antihyperglycemic effects of Elaeocarpus ganitrus roxb (Rudraksha) in streptozotocin-induced diabetes. Int J Pharm Pharm Sci 2015;7:280-3.

8. Irfan AK, Atiya K. Herbal Medicine for Diseases. Germany: India Lambert Academic Publications; 2005.

9. Petchi RR, Vijaya C, Devika GS. Evaluation of the anti-diabetic activity of Cassia surattensis Burm. Flower in streptozotocin-induced diabetic rats. Int J Res Pharm Sci 2012;2:200-5.

10. El-Sawi SA, Sleem AA. Antihyperlipidemic, antihyperglycemic and chemical composition of Senna surattensis (Burm.f.) leaves. Can J Pure Appl Sci 2009;3:779-85.

11. El-Sawi SA, Sleem AA. Flavonoids and hepatoprotective activity of leaves of Senna surattensis (Burm.f.) in CCl4 induced hepatotoxicity in rats. Can J Pure Appl Sci 2009;3:779-85.

12. Sangetha SN, Zuraini Z, Sasidharan S, Suryani S. Antimicrobial activities of Cassia surattensis and Cassia fistula. J Mol Biol Biotechnol 2008;1:1-4.

13. Sangethaa S, Sasidharanb S, Zurainia Z, Suryania S. Antioxidant activity of methanolic extracts of Cassia surattensis. Pharmacologyonline 2008;2:829-38.

14. Ellappan T, Parimaladevi B, Kumarappan C, Mandal SC. $\alpha$-glucosidase and $\alpha$-amylase inhibitory activity of Senna surattensis. J Acupunct Meridian Stud 2013;6:24-30.

15. Evans, WC. Trease and Evans Pharmacognosy. $15^{\text {th }}$ ed. London: W.B. Sanders; 2002. p. 183-393.

16. Kumarappan CT, Nageswara RT, Mandal SC. Polyphenolic extract of Ichnocarpus frutescens modifies hyperlipidemia status in diabetic rats. J Cell Mol Biol 2007;6:175-87.

17. Sumathy V, Zakaria Z, Sasidharan S. In vivo toxicity study of Cassia surattensis flower extract. Res J Pharm Biol Chem Sci 2001;2:607-17.

18. Kumar US, Chen Y, Kanwar JR, Sasidharan S. Redox control of antioxidant and antihepatotoxic activities of Cassia surattensis seed extract against paracetamol intoxication in mice: In vitro and in vivo studies of herbal green antioxidant. Oxid Med Cell Longev 2016;2016:6841348

19. Lenzen S. The mechanisms of alloxan-and streptozotocin-induced diabetes. Diabetologia 2008;51:216-26.

20. Okoro IO, Umar IA, Atawodi SE, Anigo KM. In vitro and in vivo antihyperglycemic effect of an active fraction of Cleome rutidosperma DC. Int J Pharm Pharm Sci 2015;7:289-95.

21. Meghrani M, Lemhadri A, Zeggagh NA, El Amraoui M, Halou M, Jouad H, et al. Effects of an aqueous extract of Triticum repens on lipid metabolism in normal and recent-onset diabetic rats. J Ethnopharmacol 2004:90:331-7.

22. Lemhadri A, Hajji L, Miche, JB, Eddouks M. Cholesterol and triglycerides lowering activities of caraway fruits in normal and streptozotocin diabetic rats. J Ethnopharmacol 2006;106:321-6.

23. Selvin E, Marinopoulos S, Berkenblit G, Rami T, Brancati FL, Powe NR, et al. Meta-analysis: Glycosylated hemoglobin and cardiovascular disease in diabetes mellitus. Ann Intern Med 2004;141:421-31.

24. Saddala RR, Lavanya T, Kesireddy SR. Dosage response of Pimpinella tirupatiensis tuberous root extract on the hyperglycemic condition in stzinduced diabetic rats with references to short and long-term treatment. Int J Pharm Pharm Sci 2015;7:389-92.

25. Mahendran S, Badami S, Maithili V. Evaluation of antidiabetic effect of embelin from embelia tribes in alloxan-induced diabetes in rats. Biomed Pharmacother 2010:1:25-31.

26. Balamurugan M, Parthasarathi K, Ranganathan LS, Cooper EL. Hypothetical mode of action of earthworm extract with hepatoprotective and antioxidant properties. J Zhejiang Univ Sci B 2008;9:141-7.

27. Reddi TV, Naidu BV. Ethnomedicine and Human Welfare. India: Lambert Academic Publications; 2005. p. 53. 